\title{
Experimental and Computational Study of Archery Arrows Fletched with Straight Vanes ${ }^{\dagger}$
}

\author{
Julio Ortiz ${ }^{1, *}$, Atsushi Serino ${ }^{1}$, Toshinari Hasegawa ${ }^{1}$, Takahito Onoguchi ${ }^{1}$, Hiroki Maemukai ${ }^{1}$ \\ Takeshi Miyazaki ${ }^{1}$ and Hiroki Sugiura ${ }^{2}$ \\ 1 Department of Mechanical Engineering and Intelligent Systems, Graduate School of Informatics and \\ Engineering, University of Electro-Communications, 1-5-1 Chofugaoka, Chofu, Tokyo 182-8585, Japan; \\ s1832062@edu.cc.uec.ac.jp (A.S.); t.hasegawa@uec.ac.jp (T.H.); o1932022@edu.cc.uec.ac.jp (T.O.); \\ m1932085@edu.cc.uec.ac.jp (H.M.); miyazaki@miyazaki.mce.uec.ac.jp (T.M.) \\ 2 JAXA, 7-44-1 Jindaiji-higashimachi, Chofu, Tokyo 182-8522, Japan; sugiura.hiroki@jaxa.jp \\ * Correspondence: ortiz.julio@uec.ac.jp \\ + Presented at the 13th Conference of the International Sports Engineering Association, Online, 22-26 June 2020. \\ Published: 15 June 2020
}

\begin{abstract}
The aerodynamic characteristics of archery arrows fletched with two types of straight vanes, for which the area is different, were studied. The arrows' pitching moment $\left(C_{M}\right)$, lift $\left(C_{L}\right)$ and drag $\left(C_{D}\right)$ coefficients were measured in the $60 \times 60 \mathrm{~cm}$ Magnetic Suspension and Balance System (MSBS) from JAXA. At a Reynolds number of $\operatorname{Re}=1.2 \times 10^{4}$, the values of $C_{D}$ were 1.56 and 2.05 for the short and large vanes, respectively. In a second experimental procedure, the arrows' deceleration in free flight was measured by inserting an acceleration sensor inside their shafts. For shots with an initial velocity of around $56.4 \mathrm{~ms}^{-1}$, a velocity decay of around $8 \%$ was measured. A turbulent-laminar boundary layer transition during free flight was found for shots with an average $\operatorname{Re}=1.8 \times 10^{4}$. Lastly, through numerical computations, the area difference of the two vanes was analyzed to verify the importance of $C_{M}$ and $C_{L}$ during the arrows' flights.
\end{abstract}

Keywords: archery arrow; straight vanes; acceleration sensor; magnetic wind tunnel; numerical computations

\section{Introduction}

With the increasing popularity of sports engineering, new techniques and tools are being developed to improve the performance of sporting equipment. The present study attempts to advance the understanding of the dynamics of arrows used in archery competition.

Previously, Miyazaki [1] analyzed the influence of arrows' points in their dynamics. Two different points were studied, the so-called bulge-type and a streamlined. At a Reynolds number of around $\operatorname{Re}=2.4 \times 10^{4}$, the boundary layer appeared to be fully turbulent when using the bulge-type point. For the same Re value and using the streamlined point, laminar values of the drag coefficient, $C_{D}$, were found. The latter suggests that with a proper selection of an arrow's point, the archer may be able to control the transition from a laminar to a turbulent boundary layer. The deviations in the trajectories of arrows subject to background wind, with laminar and turbulent boundary layers, have been compared [2]. Such deviations in the trajectory are clearly larger for those arrows with turbulent boundary layers than for the laminar cases.

Miyazaki [3] also studied the response of arrows fletched with two different types of curved vanes. It was concluded that by using the vanes with larger area, it is possible to improve the stabilization of arrows during their flights. Numerical computations have been performed [2] to obtain the trajectories of arrows subject to background wind in an archery range of $70 \mathrm{~m}$. Nevertheless, in such computations, $C_{D}$ was considered to remain constant along the complete 
arrows' flight. However, it is known that an arrow's velocity decays around $15 \%$ in a $70 \mathrm{~m}$ archery field [2]. Since the drag force exerted on flying arrows is proportional to the square of the velocity [3], the variation of the velocity, and likewise $C_{D}$, must be taken into account to carry out a more realistic analysis.

In this study, the changing drag force exerted on arrows during free flight was measured by an acceleration sensor inserted in the arrow shaft. The experiments were performed at a $55 \mathrm{~m}$ indoor archery range. The arrows were fletched with two types of straight vanes, for which the area is different. The static aerodynamic properties of the arrows were measured in the $60 \times 60 \mathrm{~cm}$ Magnetic Suspension and Balance System (MSBS) from JAXA. Finally, the obtained aerodynamic properties of the arrows were considered in the computation of the equations of a rigid body to simulate the trajectory and attitude of arrows in free flight. From the numerical simulations it is possible to observe some of the flight characteristics that cannot be appreciated during shooting experiments or in MSBS tests.

\section{Materials and Methods}

This section describes the arrows' characteristics, the two experimental procedures and the details of the numerical computations.

\subsection{Description of the Arrows and the Acceleration Sensor}

Arrows are elongated bodies composed of a shaft, a point and a set of fletched vanes for stabilization. The shaft used was an A/C/E $1206 \mathrm{H}$ series/1000, manufactured by wrapping an outer carbon sheet around an inner aluminum alloy tube (Figure 1a). Its mean external radius was $r=2.62$ $\times 10^{-3} \mathrm{~m}$. The point was a bulge-type point and its corresponding insert was shaped to hold steady the point with the arrow's shaft. The total length of the arrow was $l=0.638 \mathrm{~m}$. Three straight plastic vanes were fletched in the rear part of the arrow to give stabilization during flight. The chosen vanes were Easton Diamond Vanes, size 175 and size 280, hereafter referred to as short and large vanes, respectively (Figure $1 \mathrm{~b}$ ). The total mass of the arrow fletched with short vanes was $14.2 \times 10^{-3} \mathrm{~kg}$, whereas the mass for the arrow fletched with large vanes was $15.3 \times 10^{-3} \mathrm{~kg}$.

The three components of the angular velocity and the acceleration of the arrow were measured using the acceleration sensor (Logical Product, LP-UUEC002) shown in Figure 1a. It was inserted inside the arrow's shaft and located in the front part, near the point. The total mass of the sensor, holders (to fix it tightly inside the arrow's shaft) and battery was $1.37 \times 10^{-3} \mathrm{~kg}$. The acceleration sensor operated with a measurement frequency of $200 \mathrm{~Hz}$ and its acceleration range was $\pm 16 \mathrm{G}$. Its maximum rotation rate range was up to $\pm 2250 \mathrm{deg} \cdot \mathrm{s}^{-1}$.

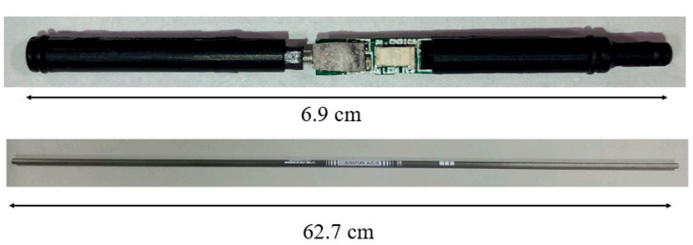

(a)

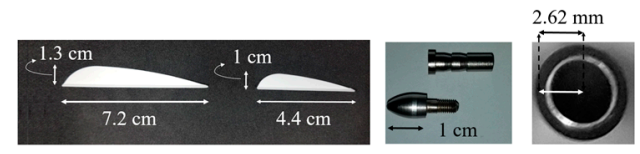

(b)

Figure 1. (a) Superior part: acceleration sensor, bottom: arrow shaft; (b) left: two straight vanes, center: screw-in point with insert, right: cross-sectional view of the arrow's shaft.

\subsection{Free-Flight and MSBS Experiments}

The free flight tests were performed at an indoor archery range of $55 \mathrm{~m}$ using a compressed-air launching system, which is a cannon-like device [1]. This propulsion system allowed us to fix the arrows in a steady position before the shot by inserting them in the cannon's nozzle. To make sure that the initial orientation of the arrows was the same during all the tests, the nozzle was introduced fully into the shaft. The compressed-air launching system also prevented the arrows from showing the characteristic flexural oscillation that would be present if the arrow were shot with 
a recurve bow. It was possible to shoot the arrows with any initial velocity, $V_{0}$, up to $75 \mathrm{~ms}^{-1}$ by adjusting the air's pressure. The pressure range was $4.5-7 \mathrm{MPa}$, which corresponds to an initial velocity range of $48-57 \mathrm{~ms}^{-1}$. All the ventilation systems were turned off to avoid the interference of the background wind. The arrows' initial velocity and initial angle of attack were obtained by recording their flights using a high-speed video camera located at $1 \mathrm{~m}$ from the launching position. The velocity was verified to decay less than $1 \%$ between the launching and the camera positions. Using the acceleration sensor, it was possible to obtain the arrows' acceleration [G] and the angular velocity [deg.s $\mathrm{s}^{-1}$ ] in the $x, y$ and $z$ components.

The MSBS is a magnetic wind tunnel that allowed us to suspend the arrows against gravity by using a magnetic field. It was possible to obtain the aerodynamic loads exerted on the arrows from the variation in the electric current necessary to keep them fixed in position. Through the MSBS tests, the values of the drag $\left(C_{D}\right)$, lift $\left(C_{L}\right)$ and pitching moment $\left(C_{M}\right)$ coefficients were obtained.

\subsection{Mathematical Model}

In this section, the mathematical model used to simulate the motion of archery arrows in free flight, the chosen numerical method to test such a model and the initial conditions used in the computations are described [3].

\subsubsection{Equations of Arrow Motion}

The arrows were assumed to be rigid bodies; therefore, no flexural oscillation along the shaft was considered. The effect of the background wind was also neglected due to the fact all the tests were performed indoors. An $x, y$ and $z$ coordinate system is defined with $i, j$ and $k$ as the corresponding unit vectors (Figure 2). The velocity of the arrow's center of mass is denoted as $V=(V$ $\sin \Theta \cos \Phi, V \sin \Theta \sin \Phi, V \cos \Theta)$, where $\Theta$ is the angle formed between $V$ and the $z$ axis. The angle $\Phi$ is formed between the $x$ axis and the projection of $V$ in the horizontal $x-y$ plane. The instantaneous velocity is $V$.

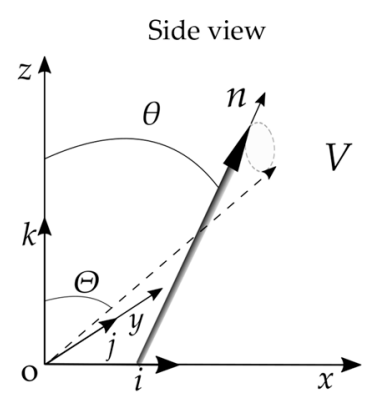

(a)

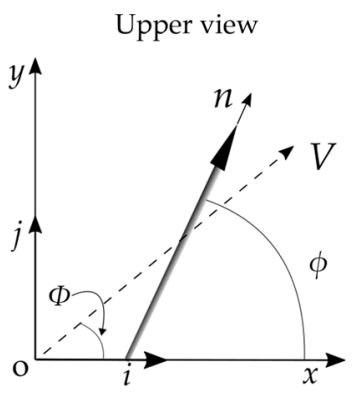

(b)

Figure 2. (a) Side and (b) upper views from an arrow in free flight.

During its free flight, the arrow's axis might not be aligned with the velocity vector $V$, as shown in Figure 2a. To measure such misalignment, the concept of angle of attack can be introduced and defined as $\gamma=\cos ^{-1}[n \cdot V /|V|]$, where $n$ is a unit vector along the arrow's axis. The lift and drag forces exerted on the arrows are expressed as $\boldsymbol{F}_{L}=\alpha \rho \pi r^{2}\left[|\boldsymbol{V}|^{2} \boldsymbol{n}-(\boldsymbol{n} \cdot \boldsymbol{V}) \boldsymbol{V}\right] / 2$ and $\boldsymbol{F}_{D}=-C_{D} \rho \pi r^{2}|\boldsymbol{V}| \boldsymbol{V} / 2$, respectively. Here $\alpha$ is a parameter related to the lift coefficient by $C_{L}=\alpha \gamma$. The air's density is $\rho$, the arrow's radius is $r$ and $C_{D}$ corresponds to the drag coefficient. The angular momentum is defined as $L=I n \times \mathrm{d} n / \mathrm{d} t+I_{3} \omega_{3} \boldsymbol{n}$, where $I$ and $I_{3}$ are the moments of inertia around the center of mass and the arrow's axis, respectively. The instantaneous value of $\omega_{3}$ was obtained from the data provided by the acceleration sensor. The equations of arrow motion are

$$
\begin{gathered}
M \frac{d V}{d t}=-M g \boldsymbol{k}+\boldsymbol{F}_{D}+\boldsymbol{F}_{L}, \\
\frac{d \boldsymbol{L}}{d t}=\operatorname{In} \times \frac{d^{2} \boldsymbol{n}}{d t^{2}}+I_{3}\left(\frac{d \omega_{3}}{d t} \boldsymbol{n}+\omega_{3} \frac{d \boldsymbol{n}}{d t}\right),
\end{gathered}
$$




$$
=\frac{1}{2} \beta \rho \pi r^{2} l|\boldsymbol{V}| \boldsymbol{n} \times \boldsymbol{V}+N_{3} \boldsymbol{n},
$$

where $\beta$ is a numerical parameter related with the pitching moment coefficient by $C_{M}=-\beta \gamma$. M is the mass of the arrow and $g$ is the gravitational acceleration. The arrow's length is given by $l$. Finally, $N_{3}$ is the axial component of the torque, $N$, exerted on the arrow during free flight and can be estimated using the data of the acceleration sensor.

\subsubsection{Numerical Computations and Initial Conditions}

From the left-hand sides of Equations (1) and (2), we have $\mathrm{d} x / \mathrm{d} t, \mathrm{~d} y / \mathrm{d} t, \mathrm{~d} z / \mathrm{d} t, \mathrm{~d} V / \mathrm{d} t, \mathrm{~d} \Theta / \mathrm{d} t$, $\mathrm{d} \Phi / \mathrm{d} t, \mathrm{~d} \omega_{\theta} / \mathrm{d} t, \mathrm{~d} \omega_{\phi} / \mathrm{d} t$, where $\mathrm{d} \theta / \mathrm{d} t=\omega_{\theta}$ and $\mathrm{d} \phi / \mathrm{d} t=\omega_{\phi}$. A standard fourth-order Runge-Kutta method was chosen to solve such a system of differential equations with a time step $\Delta t=5 \times 10^{-5} \mathrm{~s}$. The required initial conditions are given by the arrow's initial position as $x_{0}=y_{0}=0$ and $z 0=0.30 \mathrm{~m}$. The value of $z_{0}$ is the height of the launching system from the floor. The initial velocity $V_{0}$ and the initial angle between the arrow and the vertical $z$ axis, $\Theta_{0}=\theta_{0}$, were obtained from the recorded images. The values of $\Phi_{0}=\phi_{0}=0$ define that the arrow's axis is initially aligned with the target's center point. Finally, $(\mathrm{d} \theta / \mathrm{d} t)_{0}=(\mathrm{d} \phi / \mathrm{d} t)_{0}=0$ due to the arrow sliding along the nozzle of the shooting device.

\section{Results}

Figure 3a shows the dependence of $C_{D}$ on $\gamma$ for the arrows fletched with short and large vanes at $\operatorname{Re}=1.0 \times 10^{4}$, obtained from the MSBS tests. When $\gamma=0$, relatively small laminar drag coefficients were obtained. The value of $C_{D}$ increased for $\gamma \neq 0$, suggesting the transition from a laminar to a turbulent boundary layer. A typical Re for an arrow shot with a recurve bow by an archer was $\operatorname{Re} \geq 2.0 \times 10^{4}$ for which a fully turbulent flow can be expected [2]. The results in Figure 3a correspond to the half of such Re; nevertheless it can be appreciated there is a delicate dependence between $C_{D}$ and $\gamma$, even at relatively low values of Re. The drag exerted on the arrows can be said to be sensitive to the value of $\gamma$.

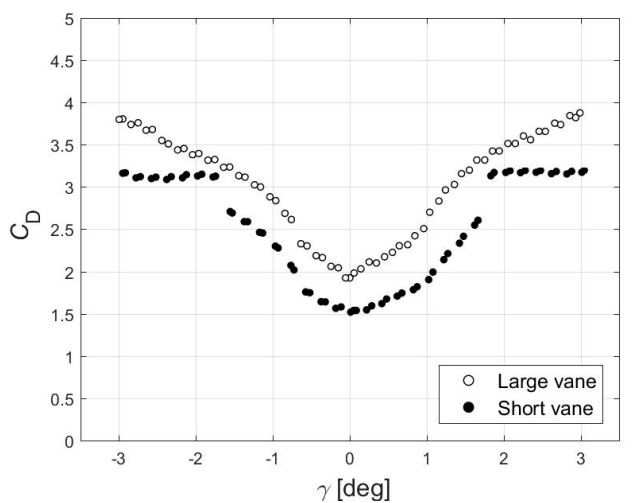

(a)

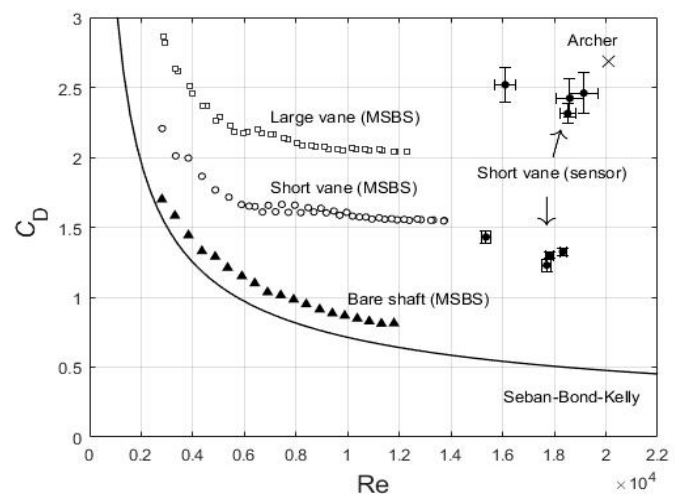

(b)

Figure 3. (a) $C_{D}$ as a function of $\gamma$ at $\operatorname{Re}=1.0 \times 10^{4}$; (b) $C_{D}$ as a function of Re obtained from the Magnetic Suspension and Balance System (MSBS) tests and the acceleration sensor.

In Figure $3 b$, the dependence of $C_{D}$ on Re obtained from the MSBS tests and the acceleration sensor is shown. The Reynolds number is given by $\mathrm{Re}=2 \mathrm{Vr} / \mathrm{vair}_{\text {, whe }}$ wair is the kinematic viscosity of the air at room temperature. The solid line shows the behavior of $C_{D}$ for a laminar boundary layer around a semi-infinite cylinder with the same radius as the analyzed arrow [4]. Closed triangles indicate the results corresponding to the bare shaft with the point attached. The difference between the bare shaft and the theoretical calculation arises from the existing pressure drag in the MSBS measurements. At $\operatorname{Re}=1.2 \times 10^{4}$, the value of the drag coefficient was $C_{D}=1.56$ and $C_{D}=2.05$ for the short (open circles) and large vanes (open squares), respectively. 
Closed circles show the results obtained from the acceleration sensor in the free flight experiments for arrows fletched with short vanes. In the Reynolds number range $1.55 \times 10^{4}<\operatorname{Re}<1.95$ $\times 10^{4}$, both laminar and turbulent values of $C_{D}$ were found, indicating a boundary layer transition. The relatively large vertical error bars suggest that a fully turbulent boundary layer is not developed for such Re. The horizontal error bars are appreciably larger for the turbulent cases than for the laminar cases, which illustrates the more rapid velocity decay, as expected. For comparison, the multiplication symbol represents the typical value for a shot performed by an elite archer equipped with a recurve bow for an A/C/E-type arrow fletched with three curved vanes. At $\operatorname{Re}=2.01 \times 10^{4}$, a turbulent value of $C_{D}=2.69$ is reported in the literature [2].

In the inferior part of Figure $4 \mathrm{a}$, we show the obtained deceleration, in the arrow's axial direction $\alpha_{\mathrm{a}}$, measured with the acceleration sensor. The case corresponds to a shot with an initial velocity of $V_{0}=56.4 \mathrm{~ms}^{-1}$ and a flying time of $1.04 \mathrm{~s}$. For $t<0.40 \mathrm{~s}$, the arrow was exposed to an average value of $0.65 \mathrm{G}$. Nevertheless, for $t>0.45 \mathrm{~s}$ the average deceleration decreased to around 0.30 $\mathrm{G}$, which can be interpreted as a transition from turbulent to laminar boundary layer during the arrow's free flight. To explain such behavior, the time evolution of the angle of attack was computed and shown in the superior part of Figure 4a. The value of $\gamma$ decayed from a maximum of around $0.36^{\circ}$ to values smaller than $0.1^{\circ}$. It is appreciated that the turbulent-laminar transition occurred when the value of the angle of attack was $\gamma<0.3^{\circ}$. The state of the boundary layer, and therefore the exerted drag, was highly sensitive to the arrows' attitude [2,3]. By integrating the deceleration, the velocity decay was found to be around $8 \%$ during free flight. Such velocity reduction might also partially explain the transition from a turbulent to a laminar boundary layer.
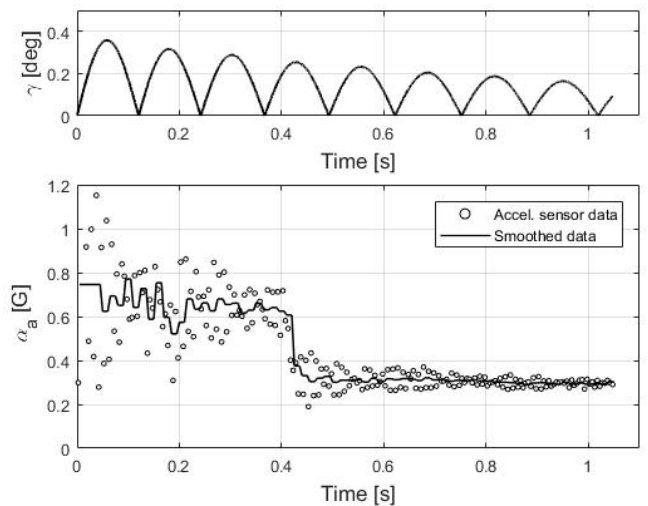

(a)

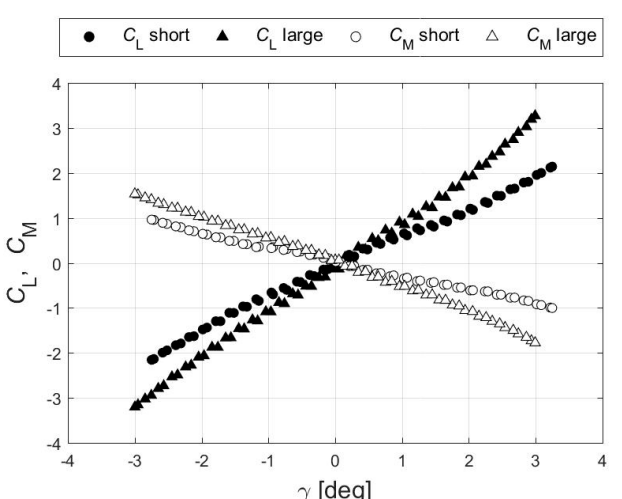

(b)

Figure 4. (a) Superior part: time evolution of $\gamma$ for a shot with initial velocity $V_{0}=56.4 \mathrm{~ms}^{-1}$, bottom: arrow's deceleration in the axial component measured with the acceleration sensor; (b) $C_{L}$ and $C_{M}$ as a function of $\gamma$ at $\operatorname{Re}=1.0 \times 10^{4}$.

Figure $4 \mathrm{~b}$ shows the lift $\left(C_{L}\right)$ and pitching moment $\left(C_{M}\right)$ coefficients as a function of $\gamma$, from which the parameters $\alpha$ and $\beta$ were obtained. $\alpha$ and $\beta$ are related with $C_{L}$ and $C_{M}$, respectively. The obtained values were $\alpha_{\text {short }}=39.1 \mathrm{rad}^{-1}$ and $\beta_{\text {short }}=18.8 \mathrm{rad}^{-1}$ for the arrows fletched with short vanes and $\alpha_{\text {large }}=62.1$ $\operatorname{rad}^{-1}$ and $\beta$ large $=33.9 \mathrm{rad}^{-1}$ for arrows fletched with large vanes. The counterbalancing effect between the lift force, exerted near the vanes, and the pitching moment, exerted around the center of mass located in the arrow's front part, has important implications. To explore the importance of the magnitude of $\alpha$ and $\beta$, the time evolution of $\gamma$ was computed for arrows with both types of vanes.

In Figure 5, the results for shots with an initial velocity of $V_{0}=56 \mathrm{~ms}^{-1}$ are shown. For the short and large vanes, respectively, the oscillation frequency of the angle of attack was $4 \mathrm{~Hz}$ and $5.3 \mathrm{~Hz}$. The larger pitching moment experienced by the arrows with large vanes also caused the oscillation frequency to increase. It was verified that for values of $\beta$ close to zero, the oscillatory arrow's behavior almost disappeared, generating large angles of attack of the order $\gamma>3^{\circ}$, and therefore increasing the exerted drag. The maximum angles of attack were $0.36^{\circ}$ and $0.27^{\circ}$ for the short and large vanes, respectively. The lift exerted on the vanes increases with their area. The larger lift forces generated by the large vanes drifted the arrow vertically and reduced the magnitude of the angle of attack. 


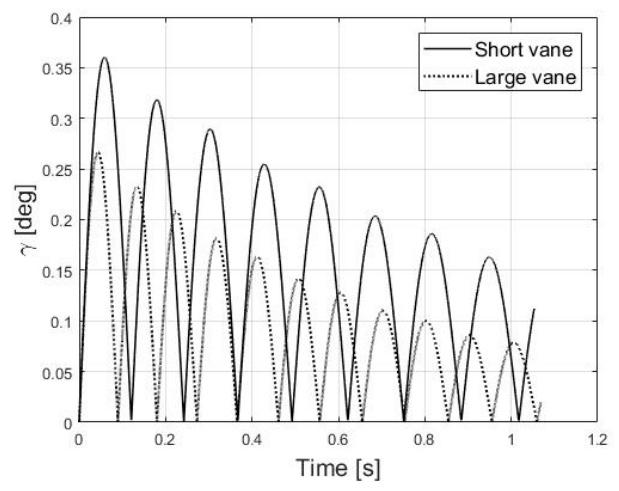

Figure 5. Time evolution of $\gamma$ for the short and large vanes with $V_{0}=56 \mathrm{~ms}^{-1}$.

\section{Conclusions}

The aerodynamic characteristics of arrows fletched with two different straight vanes, for which the area is different, were analyzed. From the MSBS tests at $\operatorname{Re}=1.2 \times 10^{4}$, the laminar values of the drag coefficient $C_{D}=1.56$ and $C_{D}=2.05$ for the short and large vanes were obtained, respectively. From the acceleration sensor data at $\operatorname{Re}<1.8 \times 10^{4}$, laminar values of around $C_{D}=1.35$ were found. For $\operatorname{Re}>1.85 \times 10^{4}$, values of the drag coefficient were around $C_{D}=2.45$, suggesting that the transition from laminar to turbulent boundary layer takes place at such Re.

A transition from turbulent to laminar boundary layer was detected with the acceleration sensor during the free flight experiments, which was explained by the fact that the value of the angle of attack reduces along the arrow's trajectory. For angles of attack $\gamma<0.3^{\circ}$, a laminar boundary layer was formed and the drag reduced in average from $0.65 \mathrm{G}$ to $0.30 \mathrm{G}$. The velocity reduction of around $8 \%$ during the flight might also contribute to the turbulent-laminar boundary layer transition.

The importance of the pitching moment and the lift exerted on the arrows during free flight was also examined. Arrows fletched with large straight vanes showed more stable flights than those fletched with the short vanes. Large values of pitching and lift moments are desirable to achieve stable flights.

Acknowledgments: This work was partially supported by a Grant in Aid for Scientific Research (C) $18 \mathrm{k} 03943$.

Conflicts of Interest: The authors declare no conflict of interest.

\section{References}

1. Miyazaki, T.; Mukaiyama, K.; Komori, Y.; Okawa, K.; Taguchi, S.; Sugiura, H. Aerodynamic properties of an archery arrow. Sports Eng. 2013, 16, 43-54. doi:10.1007/s12283-012-0102-y.

2. Ortiz, J.; Ando, M.; Murayama, K.; Miyazaki, T.; Sugiura, H. Computation of the trajectory and attitude of arrows subject to background wind. Sports Eng. 2019, 22, 7. doi:10.1007/s12283-019-0302-9.

3. Miyazaki, T.; Matsumoto, T.; Ando, R.; Ortiz, J.; Sugiura, H. Indeterminacy of drag exerted on an arrow in free flight: Arrow attitude and laminar-turbulent transition. Europhysics 2017, 38, 6. doi:10.1088/1361-6404/aa8339.

4. Seban, R.A.; Bond, R. Skin-friction and heat-transfer characteristics of a laminar boundary layer on a cylinder in axial incompressible flow. J Aeronaut Sci. 1951, 18, 671-675. doi:10.2514/8.2076.

(C) 2020 by the authors. Licensee MDPI, Basel, Switzerland. This article is an open access article distributed under the terms and conditions of the Creative Commons Attribution (CC BY) license (http://creativecommons.org/licenses/by/4.0/). 